Article

\title{
On the Use of Satellite Imagery and GIS Tools to Detect and Characterize the Urbanization around Heritage Sites: The Case Studies of the Catacombs of Mustafa Kamel in Alexandria, Egypt and the Aragonese Castle in Baia, Italy
}

\author{
Abdelaziz Elfadaly ${ }^{1,2}$ and Rosa Lasaponara ${ }^{1, *}$ \\ 1 Italian National Research Council, IMAA C.da Santa Loja, Tito Scalo, 85050 Potenza, Italy; \\ abdelaziz.elfadaly@unibas.it \\ 2 Department of Population Studies and Archaeology, National Authority for Remote Sensing and \\ Space Sciences, Cairo 1564, Egypt \\ * Correspondence: rosa.lasaponara@imaa.cnr.it; Tel.: +39-328-627-1131
}

Received: 1 February 2019; Accepted: 27 March 2019; Published: 9 April 2019

check for updates

\begin{abstract}
The sustainable development of urban growth is a mandatory challenge to be addressed, as also highlighted in the Agenda 2030, and this requires suitable and sustainable planning strategies, as well as systematic and timely monitoring of urban expansion and its effects. In this context, satellite data (today also available free of charge) can provide both (i) historical time-series datasets, and (ii) timely updated information related to the current urban spatial structure and city edges, as well as parameters to assess urban features and their statistical characterization to better understand and manage the phenomenon. Nevertheless, it is important to highlight that the identification and mapping of urban areas is still today a complex challenge, due to the heterogeneities of materials, complexity of the features, etc. Our approach, herein adopted, addresses the challenges in using heterogeneous data from multiple data sources for change detection analysis to improve knowledge and monitoring of landscape over time with a specific focus on urban sprawl and land-use change around cultural properties and archaeological areas. Two significant test cases were selected: (i) one in Egypt, the Catacombs of Mustafa Kamel in Alexandria, and (ii) one in Italy, the Aragonese Castle in Baia-Naples. For both study areas, the changes in urban layers were identified over time from satellite data and investigated using spatial analytic tools to statistically characterize them. The results of this study showed that (i) the increase in urban areas is the main phenomenon around both heritage areas, (ii) this increase is sharper in developing countries (e.g., Egypt) than developed countries (e.g., Italy), (iii) the methodology herein adopted is suitable for both big and small urban changes as observed around the Catacombs of Mustafa Kamel and the Aragonese Castle.
\end{abstract}

Keywords: urban sprawling; heritage management; free data; geographic information system (GIS); satellite images

\section{Introduction}

Over the last few years, many archaeological sites suffered from many environmental risks due to unplanned urbanization [1-6]. This study provides a short overview of space-based tools today available for urban areas ranging from planning strategies to systematic monitoring activities. In the last few decades, a rapid urbanization process occurred on the global scale, and it is still today increasing. Actually, over half of the world's population is living in urban areas and this is expected to further increase to $70 \%$ by 2050 . Therefore, the sustainable development of urban growth is a 
mandatory challenge to be addressed, and this requires suitable and sustainable planning strategies, as well as systematic and timely monitoring of urban expansion and effects. To this aim, the availability of reliable information on past and current conditions is a critical point for defining and planning potential future scenarios.

Since the early applications of Earth observation around 50 years ago, the mapping of cities and towns has been a critical challenge to address. In the 1970s, the availability of Landsat Multispectral Scanner System (MSS) pushed the early mapping of broad-scale changes at the urban-rural fringe. From the processing point of view, the most commonly used approaches were mainly based on simple band ratios, image thresholds, and image differencing [7-10]. Despite the early successful applications, the limited spatial resolution compared to aerial photographs prevented and limited the use of satellite data for urban areas. Actually, potential end-users such as decision-makers, urban planners, and land managers continued for a long time to exploit aerial photographs and field surveys [11,12].

Later, in the 1990s-2000s, the interest in global assessment of urbanization and its effects pushed the use of medium to coarse resolution [13]. Moreover, additional scientific themes such as climatology [14], hydrology [15], ecology [16], and public health [17] highlighted the importance of using satellite in a global perspective to estimate the impacts of urban expansion on environmental systems, and human health and well-being.

Subsequently, around the 2000s, the availability of very-high-resolution satellite data, such as IKONOS (1-4 m) and Quickbird (0.6-2.4 m), provided improved technical capabilities to characterize urban features with increased spatial detail $[18,19]$. Nevertheless, the cost of new acquisitions of VHR scenes, along with the sparse coverage of archived data, strongly limited once again the diffusion of satellite images for urban monitoring.

Actually, still today, medium-resolution (20-30 m) datasets such as Landsat and SPOT remain the best compromise between the availability of historical datasets and spatial detail. In particular, the Landsat Thematic Mapper (TM), Enhanced Thematic Mapper Plus (ETM+), and Operational Land Imager (OLI) satellite systems were and are the most widely used tools. Their extensive and accessible archives available for free in a wide range of environments were and are quite attractive for several urban applications. The recent availability of data from Sentinel missions opened new prospective in the field of satellite remote sensing for urban areas. Earth observation can today provide reliable tools for urban mapping from global down to a local scale by capturing the physical characteristics of urban places which generate spatial and spectral signatures. Nevertheless, it is important to highlight that the identification and mapping of urban areas is still today a complex challenge, due to the heterogeneities of features and materials, as well as the complexity and variations in size and shape of buildings and urban blocks which create mixtures within pixels.

Our approach, herein adopted, addresses the challenges in using heterogeneous information from multiple data sources for change detection analysis to improve knowledge and monitoring of landscape over time, with a specific focus on urban sprawl and land-use change around cultural properties and archaeological areas. In the last few decades, the rapid urbanization/urban redevelopment initiatives globally observed posed real challenges and opportunities for cultural heritage. Urban change can be a serious threat to cultural properties and landscape, for example, (i) urban sprawl erases the history of places, and (ii) the increase in urban areas induces an increase in atmospheric pollution and this, in turn, leads to the degradation of historical buildings, etc.

Cultural properties are particularly exposed to the negative effects of urban sprawl, considered as one of the main threats to cultural heritage; see, for example, References [1,2] (https:// whc.unesco.org/en/factors/ and http://www.unesco.org/new/en/culture/themes/culture-anddevelopment/culture-for-sustainable-urban-development/). Moreover, "the 2030 Agenda's 17 Sustainable Development Goals, SDG 11, on sustainable cities makes it clear that culture has an essential role to play in realizing sustainable urban development, particularly through strengthened efforts to protect and safeguard the world's cultural and natural heritage" (http:/ /www.unesco.org/ new/en/culture/themes/culture-and-development/culture-for-sustainable-urban-development/). 
To face this challenge, updated maps and related information are very important for urban planners to design (urban and infrastructure) development plans sensitive to cultural heritage sites. In particular, the use of satellite technologies today can suitably support the development planning process, providing suitable free-of-charge tools useful for multitemporal analysis based on the past and the current conditions, capturing changes at diverse temporal and spatial scales from global down to a local level.

Our effort is a contribution to the definition of the best practices and experiences regarding the production of critical information necessary for the preservation of cultural heritage and the necessary redevelopment of urban areas. Our approach is based on the joint use of (i) historical and updated data available from satellite technologies at a global scale free of charge, and (ii) data processing to extract useful information from the investigated dataset. In particular, the use of statistical analysis allowed us (i) to perform multiscale investigations of changes in urban areas in terms of both size and distribution, and (ii) to better capture and characterize the impact of changes delimiting urban sprawl boundaries, generally regarded as a key indicator (useful for policy measure) to control chaotic and sparse urban expansion. In other words, the use of diverse static indicators, described in Section 2, are herein proposed as tools to detect, compare, and capture the impacts of urban sprawl over time in a quantitative, objective, and reliable way for diverse case studies as in the test areas we selected (see Section 2), representative of diverse ecosystems and geographic areas in Mediterranean towns in Europe, as in the case of the Italian case study, and a desert setting in Africa, as in the case of the Egyptian area.

\section{Materials and Methods}

\subsection{Study Area}

The Roman historian Diodorus Siculus described Alexandria city (north of Egypt) as "the first civilized city in all over the ancient world" [20]. The dates of Mostafa Kamel tombs return to the late third and early second century before Christ (BC), the Greco-Roman era [21]. Mustafa Kamel Necropolis lies to the northeast of Alexandra; this Necropolis lies about 150-200 m from the seashore, and it is higher in topography than El-Shatbi and the new bibliotheca Alexandrina area. The structure of the fist tomb includes rock-cut rooms and galleries. A broad stairway leads to a square court which surrounded by Doric semi-columns, all cut into the walls, and leads to ten rooms distributed on the four sides. On the other hand, the second tomb consists of a stairway leading to a central courtyard. To the south stand, two Doric columns exist at the entrance of the room, which has luculi on both sides. Another room, with two benches and luculi on both sides, is accessible. It was probably used for prayer. At the end is a small room, in front of which a limestone offering table coated with colored plaster in imitation of alabaster was found [22]. On the other hand, the site of Baia is considered one of the most extensive submerged archaeological sites known in Italy. The site is characterized by urban sites with residential houses, thermal baths, fisheries, and harbor buildings [23,24]. Baia Castle (Naples in southern Italy) was built in 1495, under the Aragonese monarchy, upon important remnants of a Roman villa. Presently, it hosts the Campi Flegrei Archaeological Museum [25] (Figure 1a). In this paper, we exploited the integration of remote sensing, geographic information system (GIS), and statistical analyses for the identification of changes and mapping of planned and unplanned urban construction. To this aim, the datasets described in Table 1 were used for the analyses conducted in study areas selected in Italy and Egypt.

The change detection we performed was based on the use of a heterogeneous optical imagery dataset, made up Landsat TM satellite images and the more recent Sentinel 2, freely available from the United States Geological Survey (USGS) European Space Agency (ESA) web site, respectively. The approach we adopted consisted of several steps, as shown in Figure 1b, based on the multitemporal analysis of satellite data, suitably preprocessed as in Reference [1], with particular reference to 
(i) pre-processing, (ii) classification for the multidate analysis of urban expansion, and (iii) statistical analyses of the multidate maps obtained from the satellite based categorization.

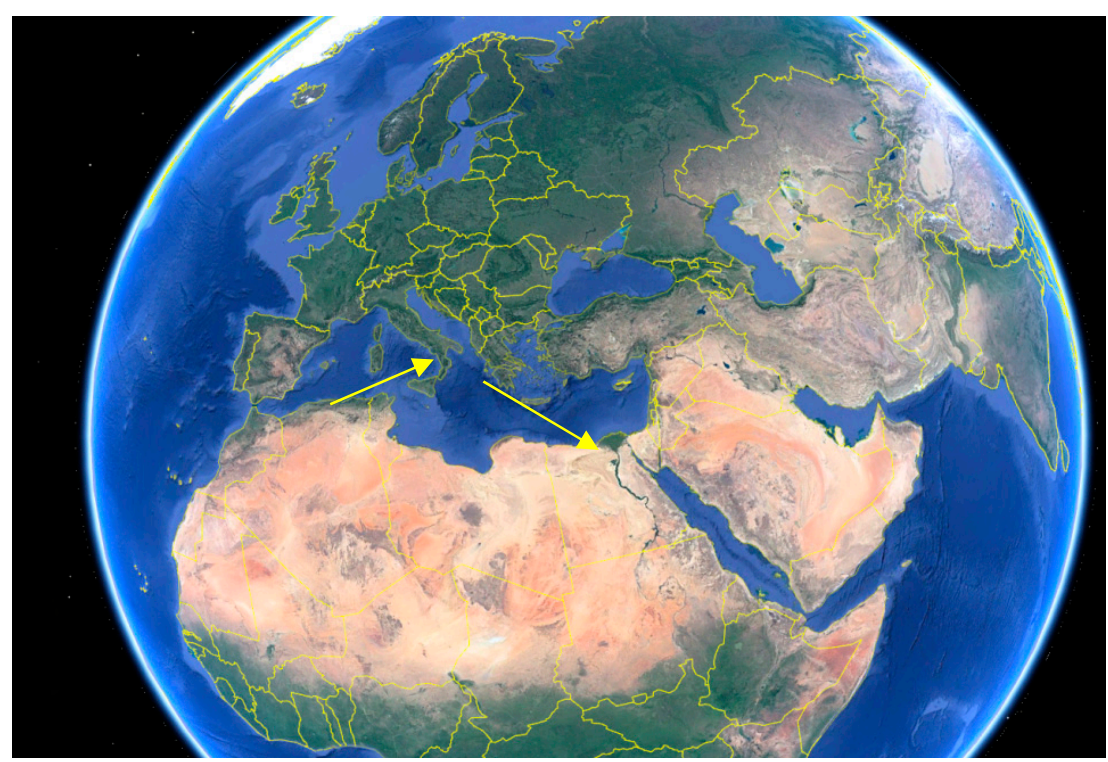

(a)

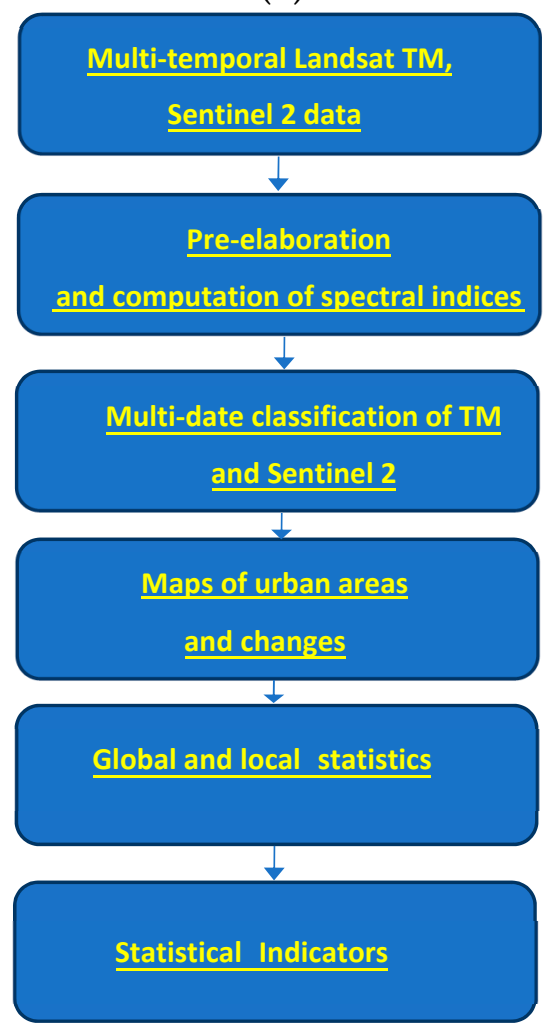

(b)

Figure 1. (a) Locations of the study areas Google Earth Courtesy, indicated by yellow arrows: Aragonese Castle in Baia (Italy) and Catacombs of Mustafa Kamel in Alexandria (Egypt); (b) flowchart of the methodological approach. 
Table 1. Main satellite images properties.

\begin{tabular}{cccccc}
\hline Number & Satellite & Sensor & Resolution (M) & Acquisition Date & Source \\
\hline 1 & Landsat & TM4 & $30 \mathrm{~m}$ & July 1998, 1999 & USGS \\
2 & Landsat & TM5 & $30 \mathrm{~m}$ & July 2008 & USGS \\
3 & Sentinel & 2A, 2B & $10 \mathrm{~m}$ & July 2018 & ESA \\
\hline
\end{tabular}

In particular, we adopted (i) unsupervised classification for the identification of the prevailing classes and their statistical distribution, (ii) supervised classification for the detection of urban areas, (iii) comparison of the outputs from the diverse images acquired in diverse years for the extraction and mapping of ongoing environmental changes with particular reference to urban sprawl, and (iv) statistical analysis. After the categorization step, for the urban layer, statistical analyses were applied in order (i) to perform multiscale investigations of changes in urban area in terms of both size and distribution, and (ii) to better capture and characterize the impact of changes delimiting urban sprawl boundaries, generally regarded as a key indicator (useful for policy measure) to control chaotic and sparse urban expansion. To this aim, spatial analyst tools were used to identify (i) statistically significant spatial clusters of high values (hot spots) and low values (cold spots), and (ii) the appropriate scale of analysis. To assess the spatial dependence, the following tests were adopted: (i) local Getis-Ord Gi* statistic, (ii) Local Moran's I statistic, (iii) Ripley's K function, and (iv) Global Moran's I, which gives five values: Moran's I index, expected index, variance, $z$-score, and $p$-value. These values evaluate whether the pattern expressed is clustered, dispersed, or random [26].

\subsection{Optimized Hotspot Analysis}

The spatio-temporal hotspot detection was based on the use of Getis-Ord Gi* statistic to statistically characterize and capture hot, non-significant, and cold spots. It is regarded as a useful tool for supporting monitoring activities, early warning, and sustainable management [27] strategies. In more detail, the optimized hotspot analysis identified statistically significant spatial clusters of high values and low values using the Gi_Bin field to identify statistically significant hot and cold spots, for multiple testing and spatial dependence using the false discovery rate (FDR) method. Features in the $+/-3$ bins (features with a Gi_Bin value of either +3 or -3 ) were statistically significant at the $99 \%$ confidence level; the clustering for features with 0 for the Gi_Bin field was not statistically significant, and the features in the $+/-2$ bins reflected a $95 \%$ confidence level; features in the $+/-1$ bins reflected a $90 \%$ confidence level [28].

\subsection{Cluster and Outlier Analysis (Anselin Local Moran's I)}

Anselin Local Moran's I statistic [29] identifies statistically significant hot spots, cold spots, and spatial outliers. This tool creates a new output feature class with Local Moran's I index, $z$-score, $p$-value, and cluster/outlier type (COType). Feature by feature, the $z$-scores and $p$-values are measures of statistical significance which inform us whether or not to reject the null hypothesis. Furthermore, this tool indicates whether the apparent similarity (a spatial clustering of either high or low values) or dissimilarity (a spatial outlier) is more pronounced than one would expect in a random distribution. In more detail, a high positive $z$-score for a feature indicates that the surrounding features have similar values (either high values or low values). In the same way, The COType field in the output feature class will be $\mathrm{HH}$ for a statistically significant cluster of high values and LL for a statistically significant cluster of low values. A low negative $z$-score (for example, less than -3.96) for a feature indicates a statistically significant spatial data outlier. On the other hand, the COType field in the output feature class will indicate if the feature has a high value and is surrounded by features with low values (HL) or if the feature has a low value and is surrounded by features with high values (LH) [30]. 


\subsection{Multi-Distance Spatial Cluster Analysis (Ripley's K Function)}

Ripley's K function definition is a tool which characterizes the spatial structure of a point or polygon patterns by graph. It is widely used in urban and vegetation studies by testing its points or polygon values against a null hypothesis. The test returns the $p$-value to reject the null hypothesis of independence between point or polygon locations [31]. The K Function Graphic Usage tool requires projected data to accurately measure distances. The tool output is a table with fields, whereby ExpectedK and ObservedK contain the expected and observed K values, respectively. When the L(d) transformation is applied, the ExpectedK values will always match the Distance value. On the other hand, the $\mathrm{K}$ function will optionally create a graph layer summarizing the results.

From the interpretation point of view, for a given distance (which denotes the scale of analysis), if the observed $\mathrm{K}$ value is larger than the expected $\mathrm{K}$ value, the distribution is more clustered than random, whereas, when the observed $\mathrm{K}$ value is smaller than the expected $\mathrm{K}$ value, the distribution is more dispersed than random distribution at that distance (scale of analysis). Nevertheless, when the observed $\mathrm{K}$ value is larger than the HiConfEnv value, the spatial clustering is statistically significant for that distance (scale). Finally, when the observed $\mathrm{K}$ value is smaller than the LwConfEnv value, spatial dispersion for that distance is statistically significant [32].

\subsection{Spatial Autocorrelation (Global Moran's I)}

Moran provided one of the first contributions to the discipline of spatial dynamics, and his index is a statistical tool useful for (i) analyzing spatial behavior, and (ii) describing how density-independent factors are correlated across wide regions [33]. In GIS software, the Spatial Autocorrelation tool gives five values: Moran's I index, expected index, variance, $z$-score, and $p$-value. This tool evaluates whether the pattern expressed is clustered, dispersed, or random. For indicating whether or not to reject the null hypothesis, this tool calculates a $z$-score and $p$-value. Optionally, when the $z$-score or $p$-value indicates statistical significance, a positive Moran's I index value indicates tendency toward clustering. On the other hand, a negative Moran's I index value indicates tendency toward dispersion. Alternatively, the null hypothesis means that the feature values are randomly distributed across the study area [34].

\section{Results and Discussion}

\subsection{Accuracy Assessment Result}

The accuracy assessment of the classification step was performed using 200 points as reference; among them, 50 points were related to urban area, 50 points were related to the Barren area, 50 points were related to vegetation, and finally 50 points were related to the water bodies. For both study areas, the classification accuracy was estimated considering these points as regions of interest (ROIs); the kappa coefficient and overall accuracy were also computed (and shown in Table 2) using a comparison with Google Earth satellite images.

Table 2. Results of kappa coefficient and overall accuracy of the regions of interest (ROIs) for each of the investigated areas between 1998 and 2018.

\begin{tabular}{ccccc}
\hline \multirow{2}{*}{ Year } & \multicolumn{2}{c}{ Alexandria Area } & \multicolumn{2}{c}{ Baia Area } \\
\cline { 2 - 5 } & Kappa Coefficient & Overall Accuracy & Kappa Coefficient & Overall Accuracy \\
\hline $\mathbf{1 9 9 8 , \mathbf { 1 9 9 9 }}$ & $96.2558 \%$ & 0.9238 & $98.8012 \%$ & 0.9739 \\
$\mathbf{2 0 0 8}$ & $96.4044 \%$ & 0.9374 & $97.1354 \%$ & 0.9395 \\
$\mathbf{2 0 1 8}$ & $92.8251 \%$ & 0.8871 & $89.8701 \%$ & 0.7896 \\
\hline
\end{tabular}


Results obtained for the Alexandria study area showed that the kappa coefficient and the overall accuracy were (i) around $96.2558 \%$ and 0.9238 , respectively, for 1999 data, (ii) around $96.4044 \%$ and 0.9374 for 2008 data, and (iii) around 092.8251 and 0.8871 in 2018.

For the study area of Baia, the kappa coefficient and the overall accuracy were around (i) $98.8012 \%$ and 0.9739 , respectively, in 1998, (ii) around $97.1354 \%$ and 0.9395 in 2008, and (iii) around 89.8701 and 0.7896 in 2018.

For both study areas, the values of the kappa coefficient and overall accuracy decreased over time due to higher spatial resolution (and in turn higher heterogeneity) of the Sentinel 2 data compared to TM.

\subsection{Change Detection}

The result from the analysis conducted using Landsat TM4 1998-1999, Landsat TM5 2008, and Sentinel-2A-B 2018 imagery revealed that, for both study areas, the urban cover increased continuously over the whole investigated period (1998 to 2018; see Table 3). In more detail, for Alexandria, the urban sprawl increased by around $1.96 \mathrm{~km}^{2}$ from 1999 to 2008 , and it increased by around $2.39 \mathrm{~km}^{2}$ from 2008 to 2018 (Figure 2). For Baia, the urban expansion increased by around $0.61 \mathrm{~km}^{2}$ from 1998 to 2008 , and it continued increasing by around $0.51 \mathrm{~km}^{2}$ from 2008 to 2018 (Figure 3).

Table 3. Total changes in urban area (expressed in $\mathrm{km}^{2}$ ) between 1998 and 2018 in Baia and Alexandria.

\begin{tabular}{|c|c|c|c|c|c|c|}
\hline Class & Study Area & $\begin{array}{c}1998,1999 \\
\left(\mathrm{~km}^{2}\right)\end{array}$ & $\begin{array}{c}\text { Change } \\
\text { Detection } \pm \mathrm{km}^{2}\end{array}$ & $2008\left(\mathrm{~km}^{2}\right)$ & $\begin{array}{c}\text { Change } \\
\text { Detection } \pm \mathrm{km}^{2}\end{array}$ & $2018\left(\mathrm{~km}^{2}\right)$ \\
\hline \multirow{4}{*}{ Urban } & Alexandria & 16.44 & 1.96 & 18.4 & 2.39 & 20.79 \\
\hline & Change \% & & $45.06 \%$ & & $54.94 \%$ & \\
\hline & Baia & 358 & 0.61 & 419 & 051 & 47 \\
\hline & Change \% & & $54.46 \%$ & & $45.54 \%$ & \\
\hline
\end{tabular}

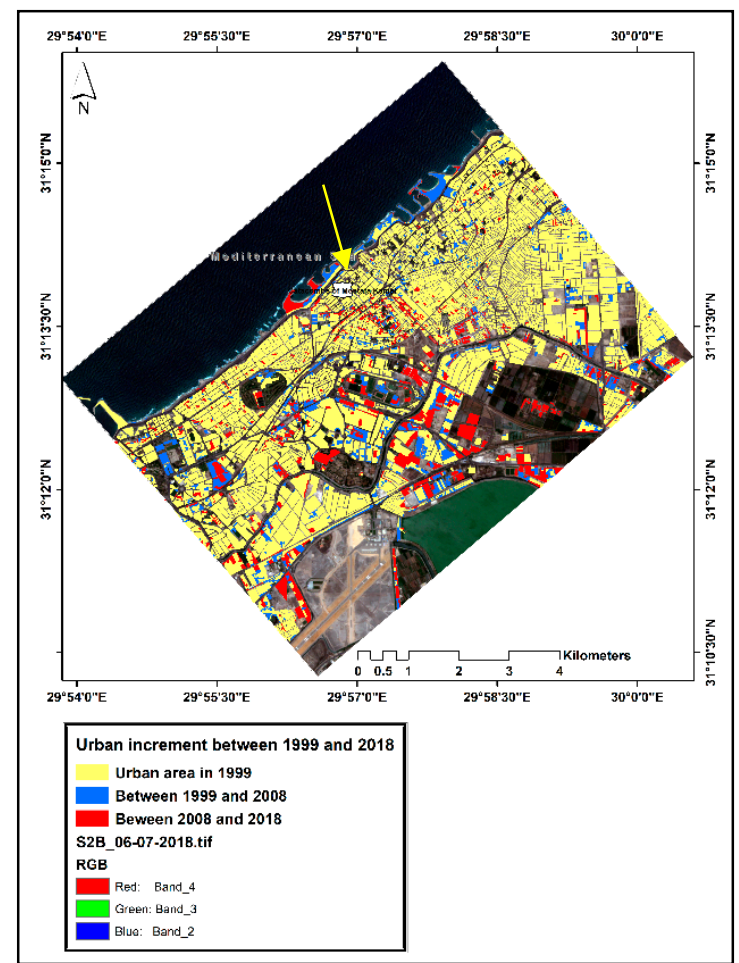

(a)

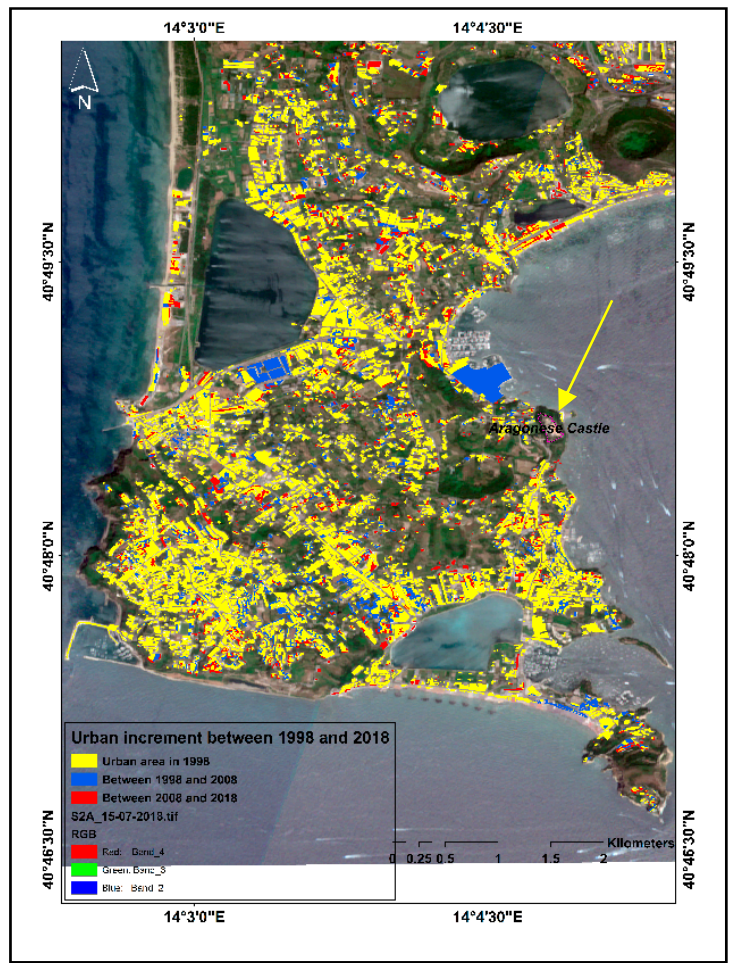

(b)

Figure 2. Changes in the urban layer between 1998 and 2018: (a) increase in urban area around the Catacombs of Mustafa Kamel (Alexandria, Egypt); (b) increase in urban area around Aragonese Castle (Baia, Naples, Italy). The yellow arrows indicate the location of the cultural properties. 


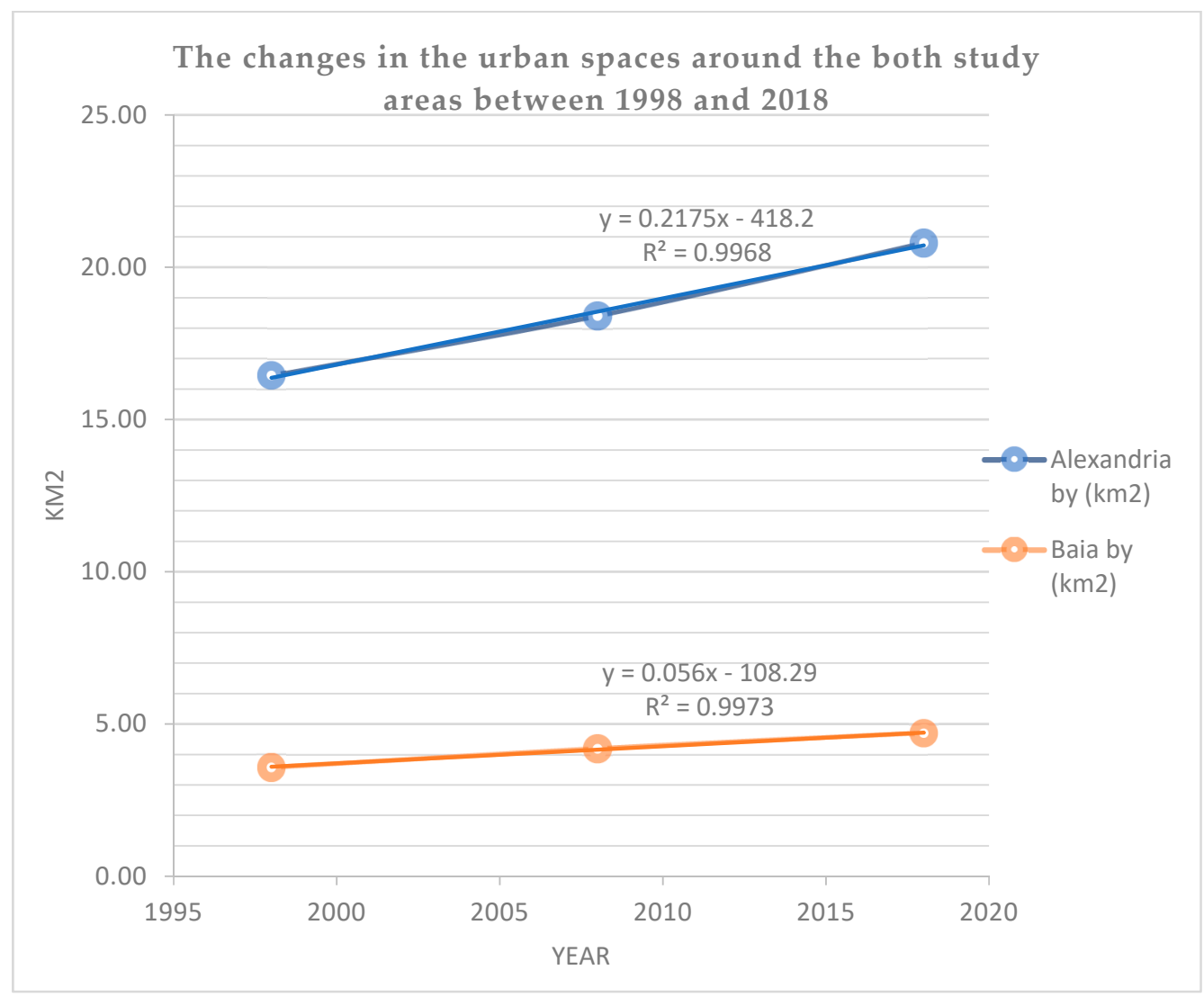

Figure 3. Graph of the increase in urban area around the Catacombs of Mustafa Kamel (Alexandria, Egypt) and Aragonese Castle (Baia, Naples, Italy).

As a whole, between 1998 and 2018 both investigated areas showed an increasing trend of urban areas; nevertheless, the changes observed for Alexandria were actually enormous and more random than those observed for the Baia case study.

\subsection{Spatial Statistcs}

The optimized hotspot analysis tool was used to calculate the pattern of spatial distribution in the urban areas of Alexandria and Baia. The Gi_Bin field identified the statistically significant hotspots, along with the non-significant and cold spots, which indicate the type of clusters for the urban layers. In the case of Alexandria, the Gi_Bin field showed that the urban space around the archaeological area was characterized by cold spots and non-significant types, whereas the hotspots were localized in the edge of the study area and especially in the south side of the heritage property in 1999 (Figure 4a).

The area of Aragonese Castle was surrounded by non-significant cluster types, whereas the cold spots were located in a small area on the north side of the heritage property, and the hotspot clusters were spread in five small areas on the north, south, and west sides in 1998 (Figure 4b).

Progressively, the situation observed for both study areas totally changed. In more detail, for Alexandria, the non-significant cluster type decreased and changed to hotspots in 2008 (Figure 5a) and similar behavior was also observed for the urban area around Aragonese Castle (Figure 5b). Furthermore, the urban areas around both culture properties moved from a non-significant type to hotspots in 2018 (Figure 6a,b) in the case of Alexandria, this trend was more clearly focused compared to Baia. 


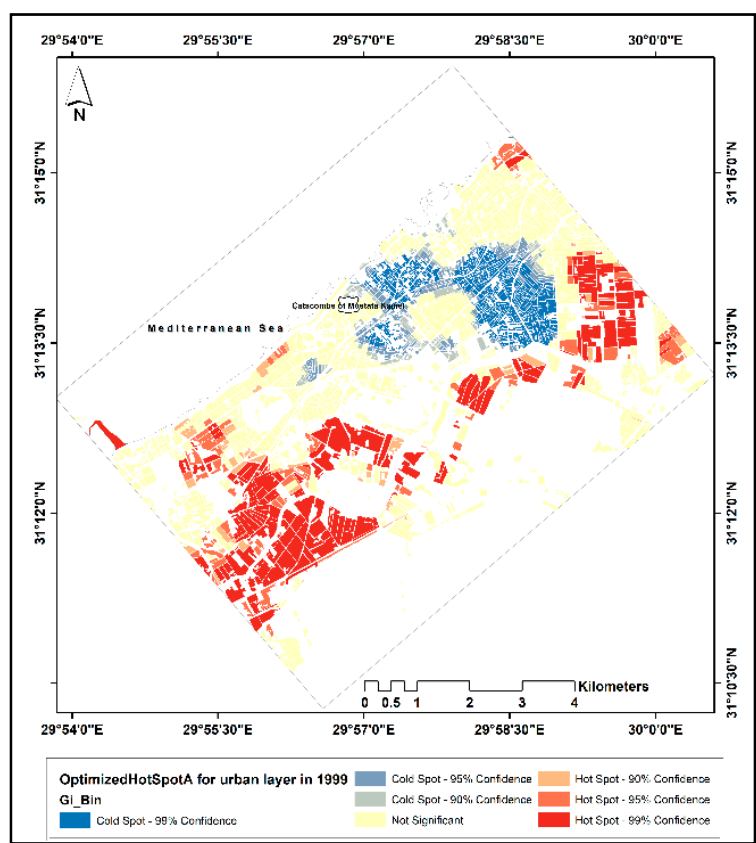

(a)

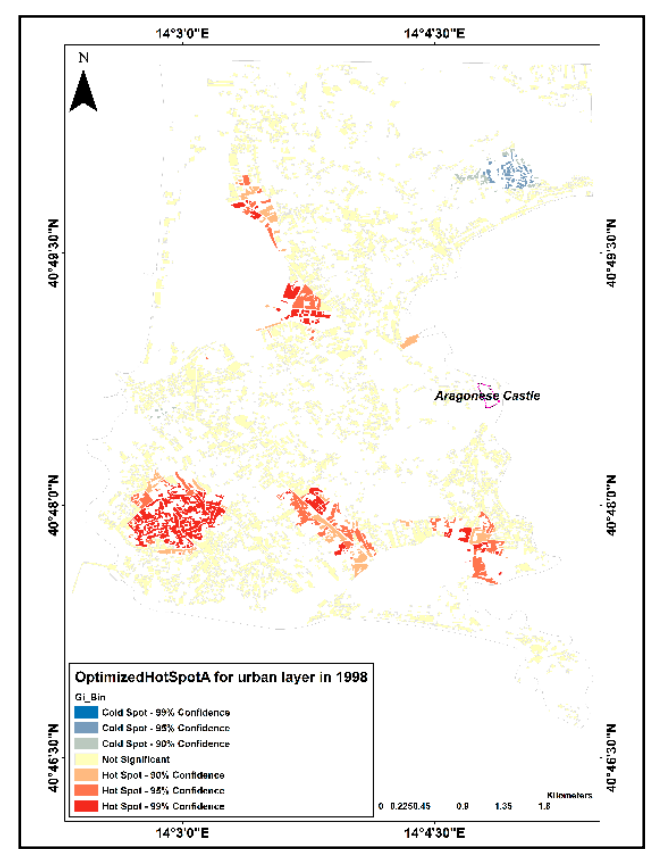

(b)

Figure 4. Levels of distribution in urban layers using the optimized hotspot tool: (a) differences between cold spot, non-significant, and hotspot levels around the archaeological area of Mustafa Kamel Necropolises in Alexandria, Egypt in 1999; (b) the open and closed spaces in the urban layer around the Castle of Aragonese in Baia, Italy in 1998.

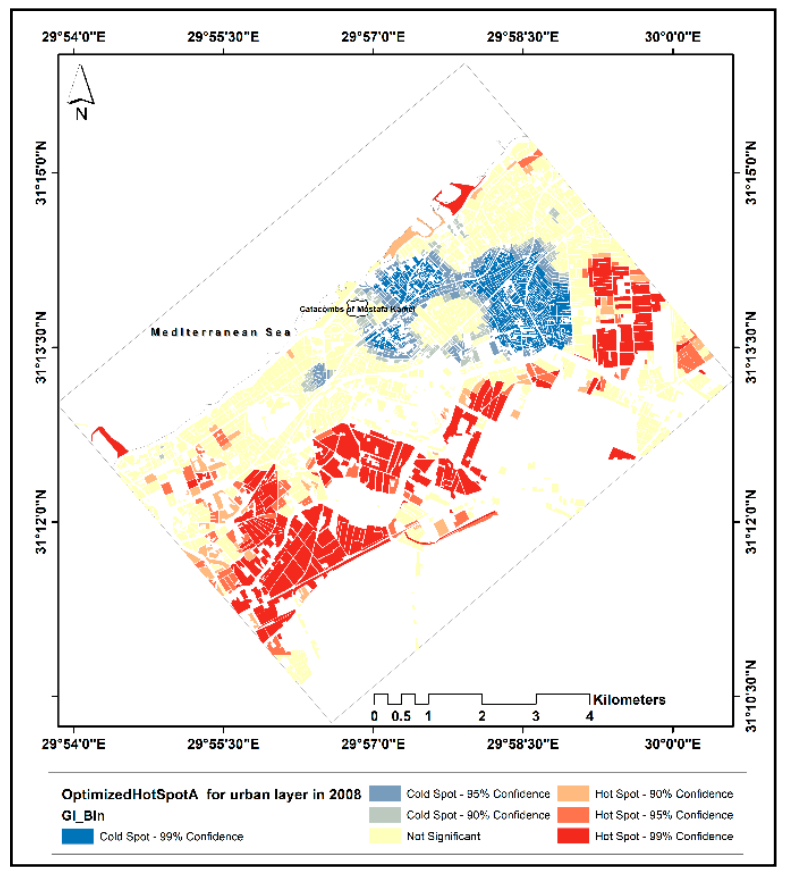

(a)

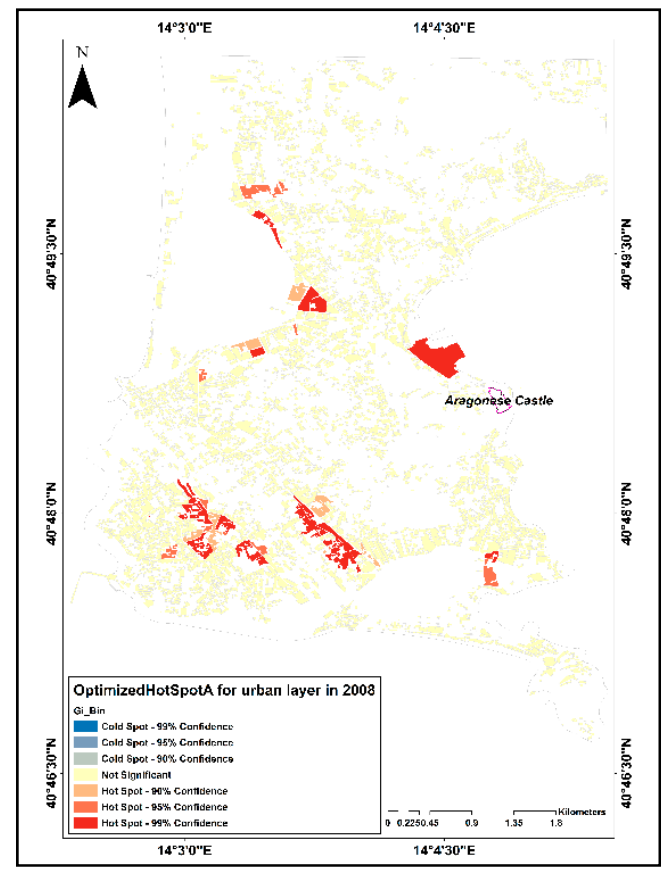

(b)

Figure 5. Urban distribution using the optimized hotspot tool in 2008: (a) differences between cold spot, non-significant, and hotspot levels around the archaeological area of Mustafa Kamel Necropolises in Alexandria, Egypt; (b) the open and closed spaces in the urban layer around the Castle of Aragonese in Baia, Italy. 


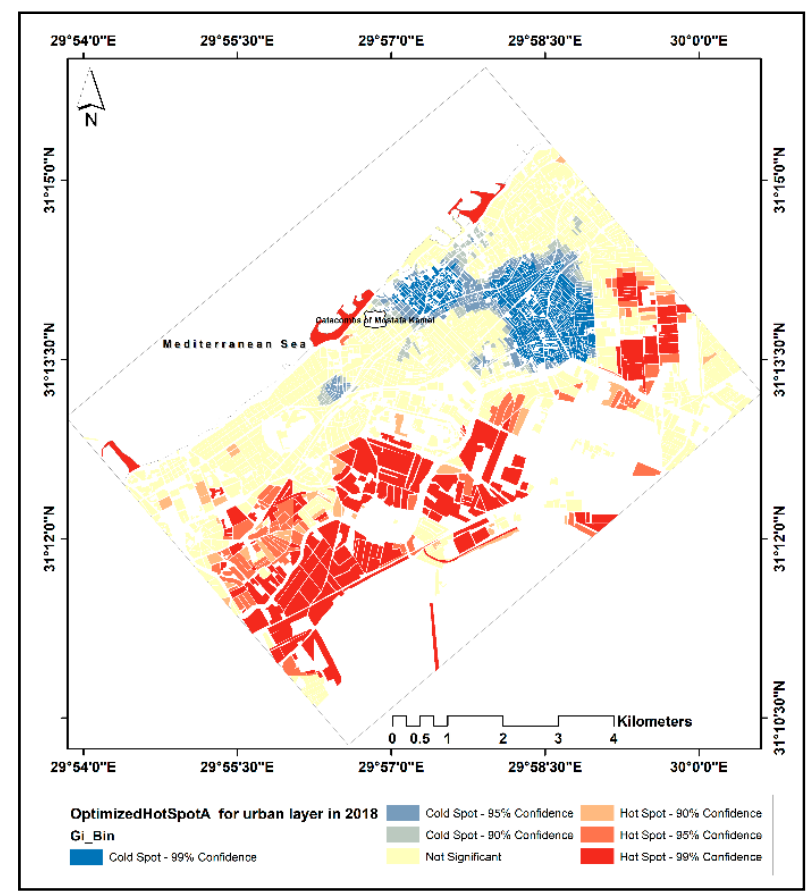

(a)

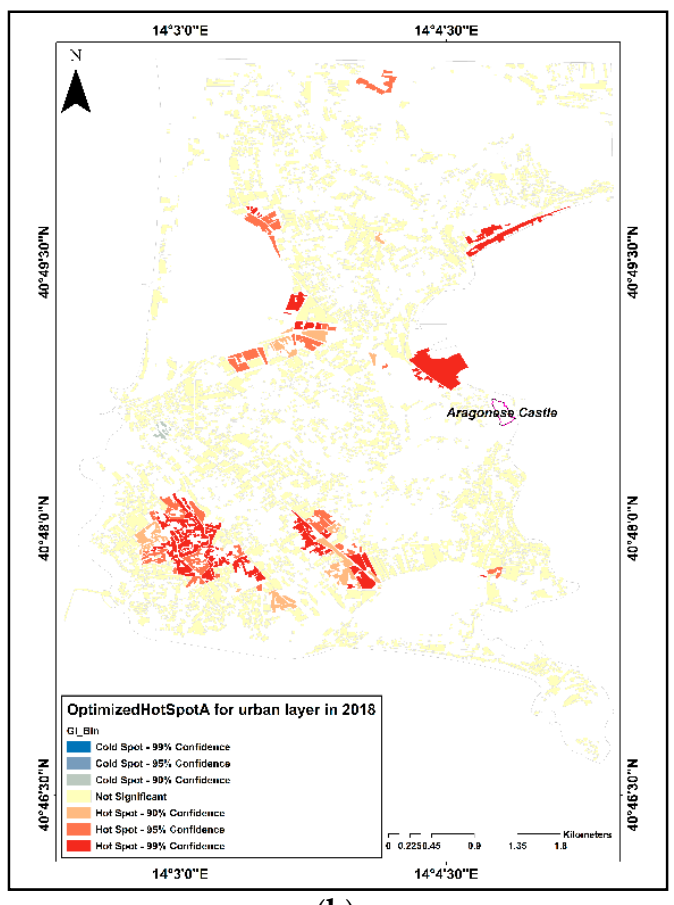

(b)

Figure 6. High levels in the hotspot areas in the urban layers using the optimized hotspot tool in 2018: (a) differences between cold spot, non-significant, and hotspot levels around the archaeological area of Mustafa Kamel Necropolises in Alexandria, Egypt; (b) the open and closed spaces in the urban layer around the Castle of Aragonese in Baia, Italy.

The optimized hotspot analysis tool was coupled with the cluster and outlier analysis (Anselin Local Moran's I) to assess the pattern of spatial distribution measuring the similarity of nearby features around the Catacombs of Mustafa Kamel in Alexandria and the Aragonese Castle in Baia.

In 1999, the cluster/outlier type (COType) results showed that the study area of Alexandria included non-significant states around the heritage area. The high/high cluster area was presented in the edge of the study area, and the high/low outlier type was spread across many points around the heritage area. Finally, the low/low cluster type appeared on the southeast side of the heritage site (Figure 7a). On the other hand, the non-significant type was the dominant status in 1998 in the Baia area, while the high/high cluster type was focused in some of the areas around the heritage area in Baia. In the same year, the high/low outlier type was presented in little areas around the heritage area (Figure 7b). Gradually, the increase in the Anselin Local Moran's I value was the dominant status in both study areas in 2008. The non-significant type decreased around the heritage area at the Catacombs of Mustafa Kamel. Furthermore, the high/high cluster area increased in Alexandria on the edge of the study area, while the high/low outlier type increased across many points near the heritage area. Finally, the low/low cluster type remained as in the previous status (Figure 8a). Concerning the Baia area in the same year, the level of increase in high/high clusters continued in the same direction, while the high/low outlier and non-significant status decreased in 2008 (Figure 8b). Furthermore, the increases in the high/high cluster and high/low outlier types were the dominant statuses in both study areas in 2018 (Figure 9a,b). 


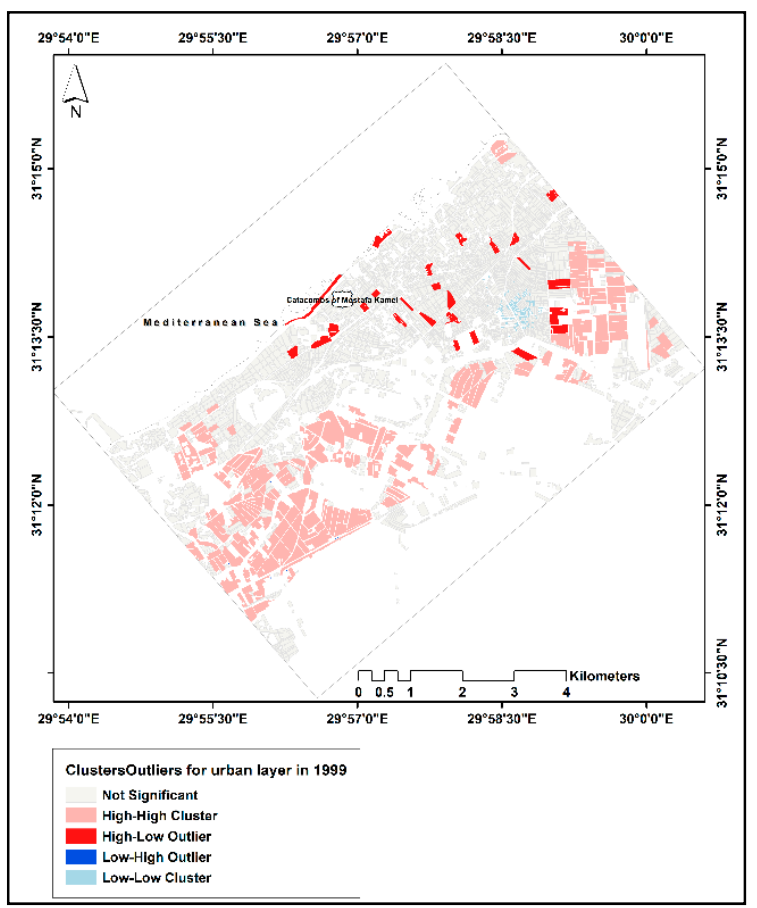

(a)

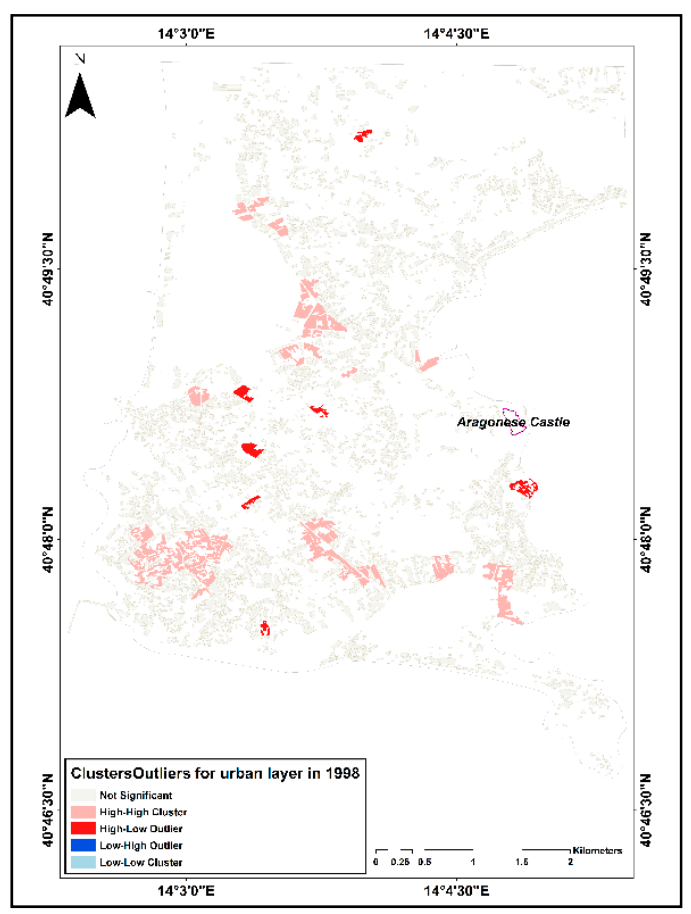

(b)

Figure 7. High and low clusters in the urban layer using the cluster outliers tool: (a) the gradient between the significant, high, and low clusters in the urban land around the heritage area of Mustafa Kamel tombs in Alexandria, Egypt in 1999; (b) the significant, high, and low levels in the urban areas around Aragonese Castle in Baia, Italy in 1998.

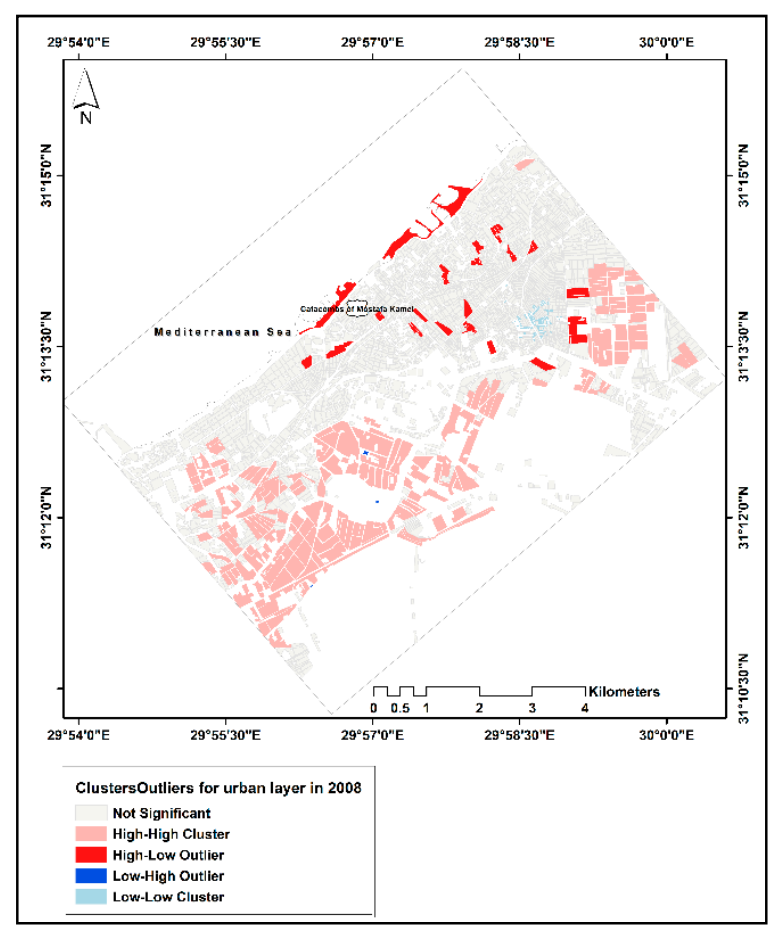

(a)

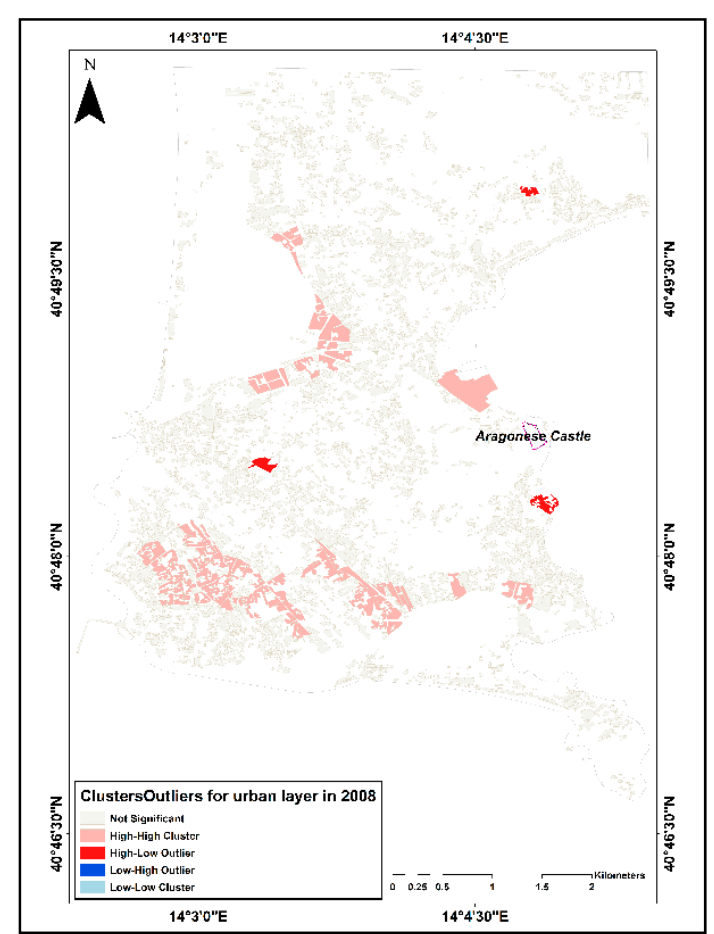

(b)

Figure 8. High and low clusters in the urban layer using the cluster outliers tool in 2008: (a) the gradient between the significant, high, and low clusters in the urban land around the heritage area of Mustafa Kamel tombs in Alexandria, Egypt; (b) the significant, high, and low levels in the urban areas around Aragonese Castle in Baia, Italy. 


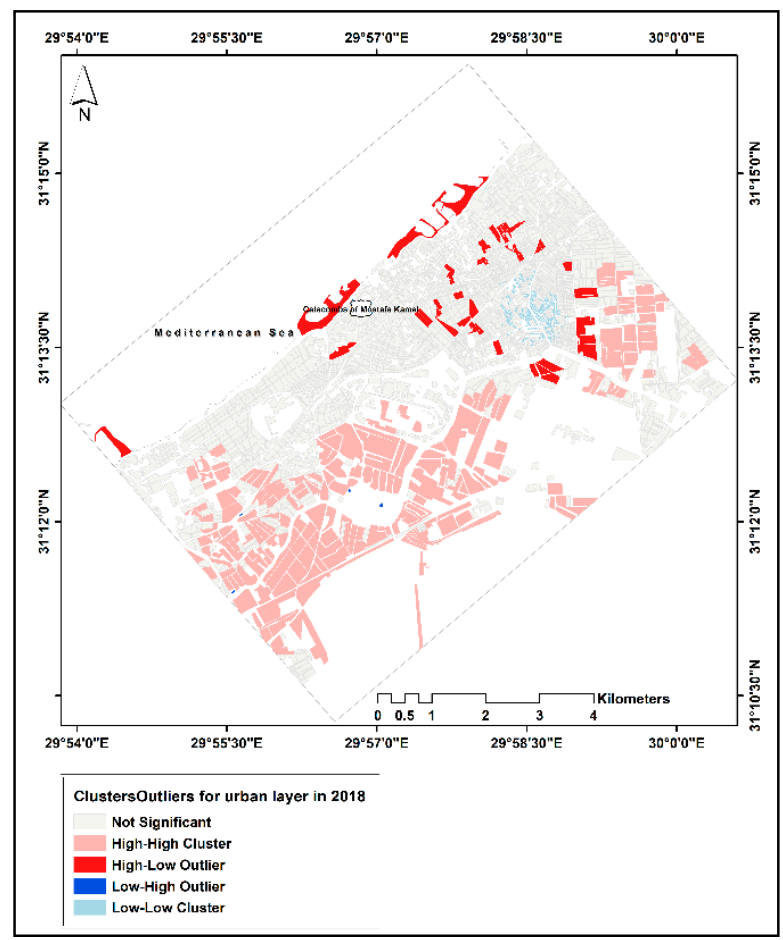

(a)

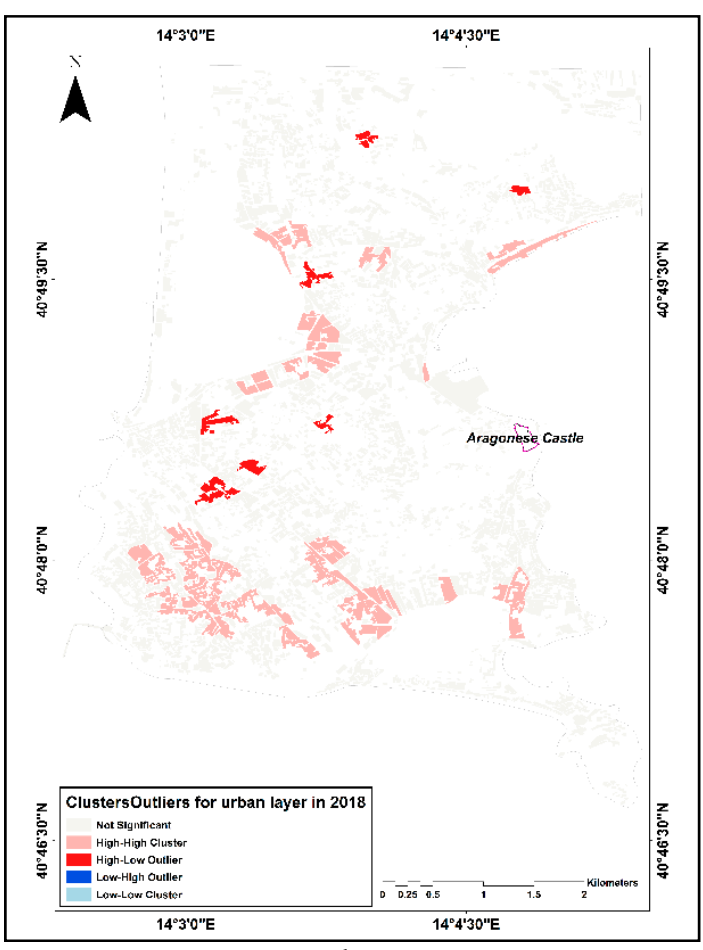

(b)

Figure 9. High and low clusters in the urban layer using the cluster outliers tool in 2018: (a) the gradient between the significant, high, and low clusters in the urban land around the heritage area of Mustafa Kamel tombs in Alexandria, Egypt; (b) the significant, high, and low levels in the urban area around Aragonese Castle in Baia, Italy.

Finally, the results obtained from both the optimized hotspot analysis (based on Getis) and outlier analysis (based on Moran) clearly pointed out that changes in the urban lands between 1998 and 2018 were more random and unplanned in the case of Alexandria than in the Baia study area.

Ripley's K function tool was used to estimate the urban distribution according to the relationship between the clustered and dispersed factors. In more detail, this tool can calculate (i) the statistically significant clustering at smaller distances, and (ii) the dispersion at larger distances for the urban distributions over the years. The results from Ripley's K function showed that the observed spatial pattern started and finished with the statistically significant dispersion at larger distances in a short space. On the other hand, there was statistically significant clustering at smaller distances in the larger space in the Alexandria area in 1999 (Figure 10a). Furthermore, the observed spatial pattern had the same status as previously with a statistically significant dispersion at larger distances in a short space. In contrast, for the highest value in the $\mathrm{L}(\mathrm{d})$ and distance, the observed spatial pattern exhibited statistically significant clustering at smaller distances in the Alexandria area in 2008 (Figure 11a). On the other hand, in 2018, the observed spatial pattern presented the status of statistically significant with clustering at smaller distances and dispersion at larger distances in the Alexandria area (Figure 12a).

In the case of Baia, the result of Ripley's K function showed that the statistically significant clustering at smaller distances presented the dominant status in 1998 (Figure 10b). In the same direction, the observed spatial pattern had statistically significant clustering at smaller distances between the clustered pattern and dispersed pattern factors in the Baia area in 2008 (Figure 11b). Furthermore, the observed spatial pattern had statistically significant clustering at smaller distances in the general view between the clustered pattern and dispersed pattern factors (Figure 12b). 


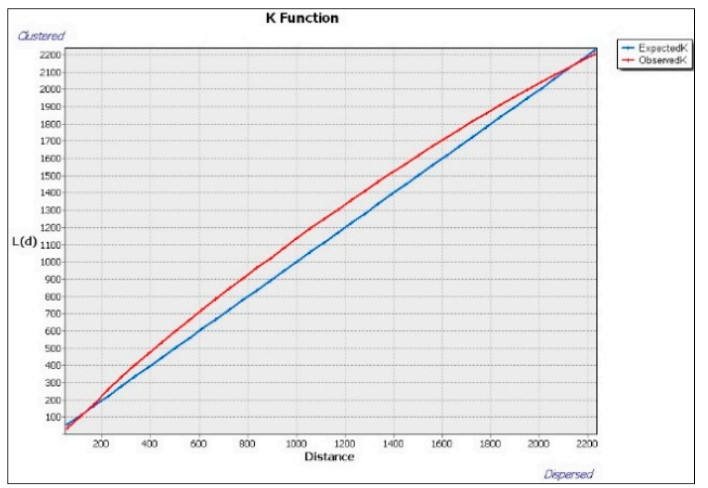

(a)

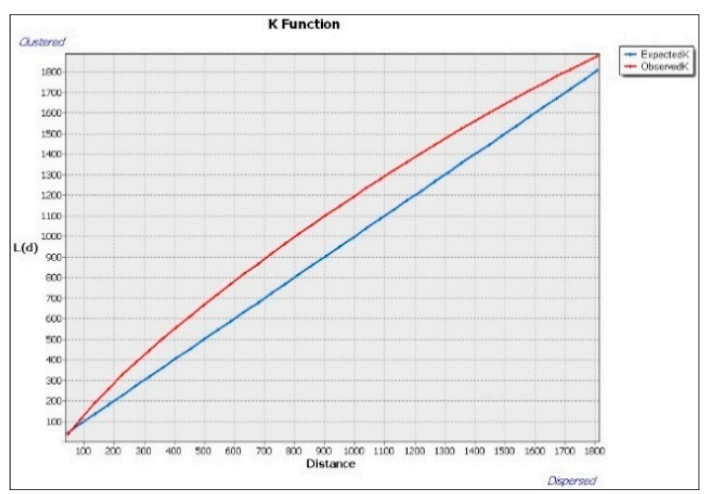

(b)

Figure 10. Relationship between the building areas and the open spaces according to the space between the red line "observed spatial pattern" and blue line "random spatial pattern": (a) K function value according to the spaces inside and between the built-up areas around the Catacombs of Mustafa Kamel, Alexandria, Egypt in 1999; (b) K function results according to the output of the built-up areas around the Aragonese Castle, Baia, Italy in 1998.

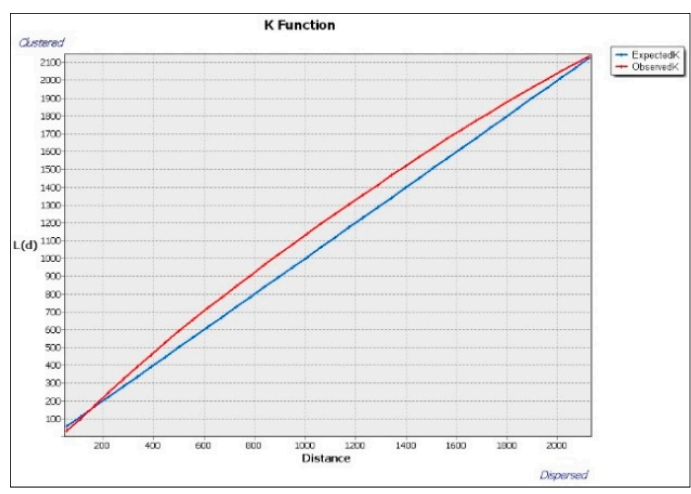

(a)

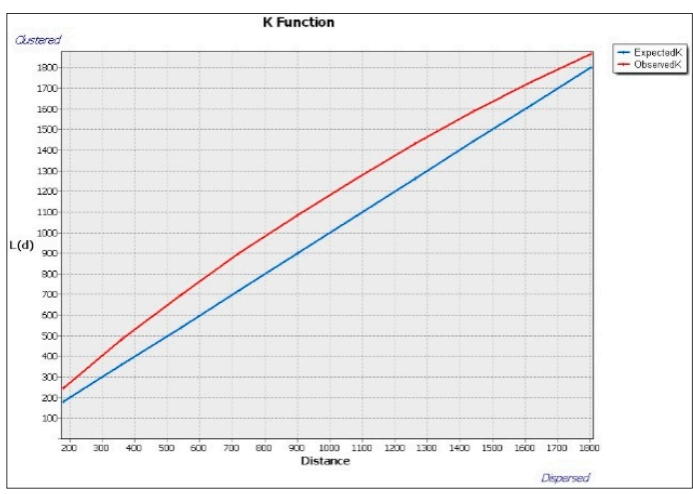

(b)

Figure 11. Relationship between the building areas and the open spaces according to the space between the red line "observed spatial pattern" and blue line "random spatial pattern" in 2008: (a) K function value according to the spaces inside and between the built-up areas around the Catacombs of Mustafa Kamel, Alexandria, Egypt; (b) K function results according to the output of the built-up areas around the Aragonese Castle, Baia, Italy.

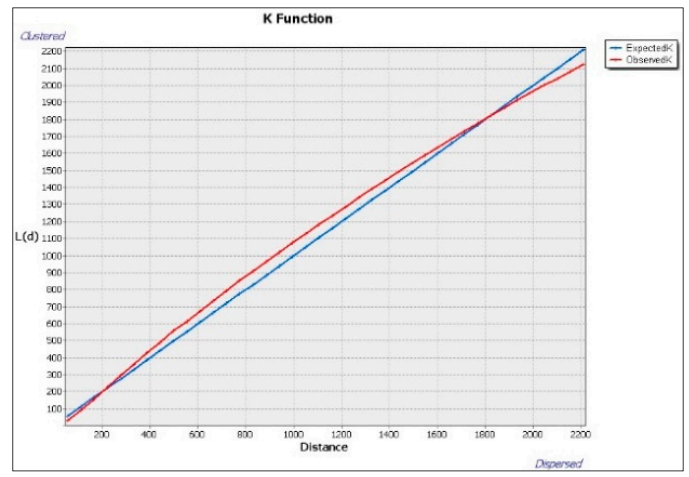

(a)

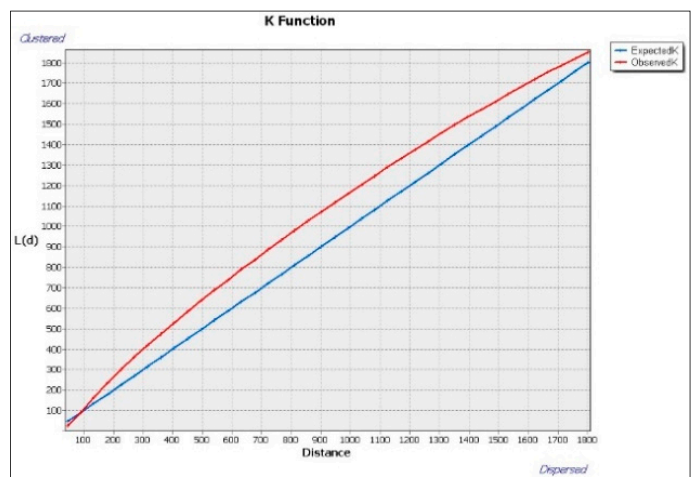

(b)

Figure 12. Relationship between the building areas and the open spaces according to the space between the red line "observed spatial pattern" and blue line "random spatial pattern" in 2018: (a) K function value according to the spaces inside and between the built-up areas around the Catacombs of Mustafa Kamel (Alexandria, Egypt); (b) K function results according to the output of the built-up areas around the Aragonese Castle at Baia (Naples, Italy). 
As a whole, according to the total results of Ripley's K function, the changes between 1998 and 2018 in the built-up areas were clearer and more random for Alexandria than Baia.

The results obtained from the pattern analyses performed using Global Moran's I for Alexandria and Baia are shown in Tables 4 and 5, respectively. The key elements for the interpretation of these tables are shown in Figure 13.

Table 4. Urban distribution patterns obtained from spatial autocorrelation for the area around the Catacombs of Mustafa Kamel between 1999 and 2018.

\begin{tabular}{cccc}
\hline Year & Moran's Index & $z$-Score & Type of Spatial Distribution \\
\hline $\mathbf{1 9 9 9}$ & 0.236786 & 66.648117 & Clustered \\
$\mathbf{2 0 0 8}$ & 0.131808 & 49.497299 & Clustered \\
$\mathbf{2 0 1 8}$ & 0.114997 & 56.709045 & Clustered \\
\hline
\end{tabular}

Table 5. Urban distribution patterns obtained from spatial autocorrelation for the area around Aragonese Castle between 1998 and 2018.

\begin{tabular}{cccc}
\hline Year & Moran's Index & $z$-Score & Type of Spatial Distribution \\
\hline $\mathbf{1 9 9 8}$ & 0.057303 & 9.354977 & Clustered \\
$\mathbf{2 0 0 8}$ & 0.051683 & 8.473026 & Clustered \\
$\mathbf{2 0 1 8}$ & 0.063031 & 10.685925 & Clustered \\
\hline
\end{tabular}

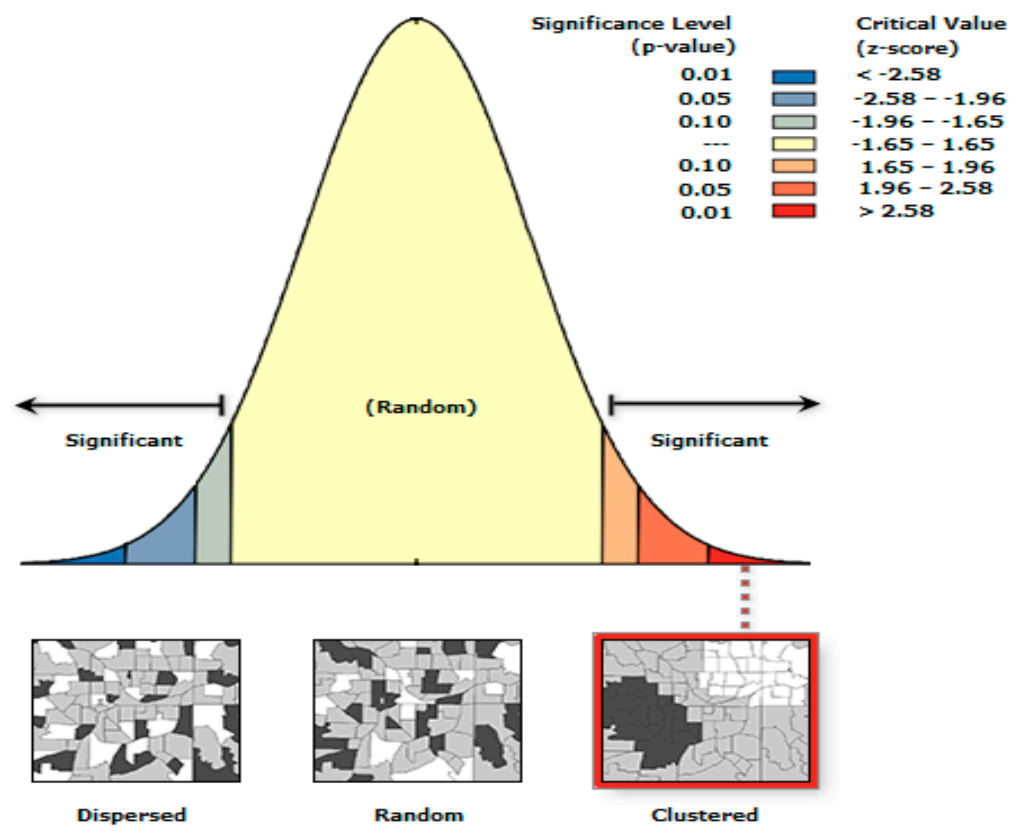

Figure 13. Graph of Global Moran's I using the spatial autocorrelation technique for the urban layer, describing the changes between the significant and random status and the gradient between the dispersed, random, and clustered status.

In particular, Table 4 shows that, for 1999, the $z$-score was around 66.64, and Moran's index was around 0.23 , and the type of spatial distribution was clustered. These values decreased in 2008 to be 49.49 for the $z$-score and 0.13 for Moran's index, while the spatial distribution had the same status as a cluster type (Table 4). In contrast, the value of $z$-score increased in 2018 to 56.7, and the value of Moran's I decreased again to 0.11 , while the spatial distribution type remained as the clustered type (Figure 13).

The result of Global Moran's I for Baia showed that z-score was 9.35, Moran's index was 0.057, and the type of spatial distribution was clustered in 1998. These values decreased in 2008 to be 8.47 for 
$z$-score and 0.051 for Moran's index, while the spatial distribution had the same status as a cluster type (Table 5). The value of $z$-score increased in 2018 to 10.68, and the value of Moran's I increased also to 0.063 , while the spatial distribution type remained as the clustered type (Figure 13).

As a whole, outputs from our investigations pointed out that the use of both global and local spatial analyses allowed us to perform multi-scale analyses of changes identified from the multi-temporal satellite-based investigations. The use of the diverse statistical indicators/tools enabled us (i) to better characterize the impact of changes over the investigated time window in terms of size and distribution, and (ii) to capture clusterization and randomness of the observed urban distribution.

In particular, results from the optimized hotspot analysis tool, used for calculating the pattern of spatial distribution, pointed out that the changes in the urban areas between 1998 and 2018 were more random and unplanned in the case of Alexandria than in the Baia study area. The optimized hotspot analysis tool was coupled with the cluster and outlier analysis (Anselin Local Moran's I) to capture clusterization and randomness of the observed urban distribution. As a whole, the optimized hotspot analysis tool indicated whether high or low values were concentrated over the study area, and cluster and outlier analysis indicated if they tended to be clustered. The results obtained from both the optimized hotspot analysis (based on Getis) and outlier analysis (based on Moran) fit each other well and coherently and clearly pointed out that changes in the urban lands between 1998 and 2018 were more random and unplanned in the case of Alexandria than in the Baia study area. The investigations were complemented by the application of Ripley's function tool, herein used for estimating the urban distribution according to the relationship between the clustered and dispersed factors. The results obtained from Ripley's function showed that the changes between 1998 and 2018 in the built-up lands were clearer and more random for Alexandria than Baia, thus confirming the trend already observed from the analysis carried out using both the optimized hotspot analysis (based on Getis) and outlier analysis (based on Moran).

Finally, the global analysis, conducted using the spatial autocorrelation statistic, quantified the impact of the change on the whole area; thus, from the variation over time, we obtained information on the compactness/randomness of the urban layer as a general global indicator.

The approach herein proposed enabled us to automatically identify and characterize the impact of changes (via the global analysis) and also to better delimit the urban sprawl boundaries (via the local analysis) and their characteristics, generally regarded as a key indicator to control chaotic and sparse urban expansion. In fact, the local analyses allowed us to assess, for example, if sprawl was due to augmented urban size or decreased urban compactness, and this can allow a practical implementation of policy and decision processes addressed to a sustainable urban development. Moreover, results from our investigations highlighted that both local and global indices were suitable for both case studies selected, as one was representative of diverse urban small towns, as in the case of the Italian case study, and one was representative of big cities, as in the case of the Egyptian area. The use of both global and local statistics enabled us to identify sprawl, applying the diverse indicators herein proposed as tools to detect, compare, and capture the impacts of urban changes over time in an automatic way.

\section{Conclusions}

The main purpose of this research was to set up low-cost and reliable tools useful for monitoring urban growth. The integration between remote sensing and GIS techniques and the joint use of analytical statistical methods for quantitative assessment enabled the identification and characterization of changes and the mapping of planned and unplanned urban construction in the two case studies selected in Italy and Egypt. Our approach addresses the challenges in using heterogeneous data from multiple data sources for change detection analysis to improve knowledge and monitoring of landscape over time, with a specific focus on urban sprawl and land-use change around cultural properties and archaeological areas. The change detection we performed was based on the use of a heterogeneous optical imagery dataset, made up of satellite Landsat TM imagery and the more recent Sentinel 2 data, freely available from the ESA web site. The approach we adopted 
consisted of (i) unsupervised classification for the identification of the prevailing classes and their statistical distribution, (ii) supervised classification for the detection of urban areas, (iii) comparison of the outputs from the diverse images acquired in diverse years for the extraction and mapping of ongoing environmental changes, with particular reference to urban sprawl. The classification accuracy, estimated for the regions of interest selected in Italy and Egypt, was higher than 98\%. Outputs from our investigations clearly highlighted that satellite data can provide very useful tools for (i) capturing land-use changes, along with impact induced by human activities at a site level, and (ii) monitoring environmental problems with a particular attention addressed to the urbanization and changes in land use/land cover in close proximity with archaeological areas and cultural property/ landscape, thereby providing reliable tools to identify changes from a global view down to a local scale.

As a whole, we can conclude that the recent availability of data from Sentinel missions opened a new prospective in the field of satellite remote sensing for urban areas. In particular, outputs from our investigations pointed out that significant improvements can be achieved from the increased spatial detail of Sentinel 2 compared to Landsat, and from the joint use of Earth observation (EO)-based geo-spatial products and geo-information. EO can provide (i) reliable analysis of current settlement patterns and their impact, with detailed information on spatial and temporal urbanization processes, and (ii) effective tools for supporting decision-makers and the future planning of urban expansion.

Author Contributions: The research article included two main contributions: GIS analysis and satellite data interpretation. The GIS analysis was carried out by A.E. Satellite data interpretation was processed by R.L. Writing of the manuscript was done through cooperation between A.E. and R.L. The last version of the article was revised by R.L.

Funding: This research received no external funding.

Acknowledgments: This article is part of the project "Detect the environmental problems around the heritage sites in Egypt and Italy due to the urban sprawling using the integration between GIS and Satellite data"; thus, the authors would like to thank the National Authority for Remote Sensing and Space Sciences (NARSS) for providing facilities for the authors. The authors would like to specially thank the Italian National Research Council (CNR) at Tito Scalo, Potenza for supporting the research activities.

Conflicts of Interest: The authors declare no conflicts of interest. The authors would also like to declare that the funding of the study was supported by the authors' institutions.

\section{References}

1. Elfadaly, A.; Attia, W.; Qelichi, M.M.; Murgante, B.; Lasaponara, R. Management of Cultural Heritage Sites Using Remote Sensing Indices and Spatial Analysis Techniques. Surv. Geophys. 2018, 39, 1347-1377.

2. Elfadaly, A.; Attia, W.; Lasaponara, R. Monitoring the Environmental Risks Around Medinet Habu and Ramesseum Temple at West Luxor, Egypt, Using Remote Sensing and GIS Techniques. J. Archaeol. Method Theory 2018, 25, 587-610. [CrossRef]

3. Elfadaly, A.; Wafa, O.; Abouarab, M.A.; Guida, A.; Spanu, P.G.; Lasaponara, R. Geo-Environmental Estimation of Land Use Changes and Its Effects on Egyptian Temples at Luxor City. ISPRS Int. J. Geoinf. 2017, 6, 378. [CrossRef]

4. Elfadaly, A.; Lasaponara, R.; Murgante, B.; Qelichi, M.M. Cultural Heritage Management Using Analysis of Satellite Images and Advanced GIS Techniques at East Luxor, Egypt and Kangavar, Iran (A Comparison Case Study). In Proceedings of the International Conference on Computational Science and Its Applications, Trieste, Italy, 2-5 July 2017; Springer: Cham, Switzerland; pp. 152-168.

5. Lasaponara, R.; Murgante, B.; Elfadaly, A.; Qelichi, M.M.; Shahraki, S.Z.; Wafa, O.; Attia, W. Spatial open data for monitoring risks and preserving archaeological areas and landscape: Case studies at Kom el Shoqafa, Egypt and Shush, Iran. Sustainability 2017, 9, 572. [CrossRef]

6. Lasaponara, R.; Elfadaly, A.; Attia, W. Low cost space technologies for operational change detection monitoring around the archaeological area of Esna-Egypt. In Proceedings of the International Conference on Computational Science and Its Applications, Beijing, China, 4-7 July 2016; Springer: Cham, Switzerland; pp. 611-621.

7. Friedman, S.Z.; Angelici, G.L. The detection of urban expansion from Landsat imagery. Remote Sens. Q. 1979, 1, 58-79. 
8. Howarth, P.J.; Boasson, E. Landsat digital enhancements for change detection in urban environments. Remote Sens. Environ. 1983, 13, 149-160. [CrossRef]

9. Jensen, J.R.; Toll, D.L. Detecting residential land-use development at the urban fringe. Photogramm. Eng. Remote Sens. 1982, 48, 629-643.

10. Todd, W.J. Urban and regional land use change detected by using Landsat data. J. Res. US Geol. Surv. 1977, 5, 529-534.

11. Michalak, W.Z. GIS in land use change analysis: Integration of remotely sensed data into GIS. Appl. Geogr. 1993, 13, 28-44. [CrossRef]

12. Ryznar, R.M.; Wagner, T.W. Using remotely sensed imagery to detect urban change: Viewing Detroit from space. J. Am. Plan. Assoc. 2001, 67, 327-336. [CrossRef]

13. Schneider, A.; Friedl, M.A.; Potere, D. A new map of global urban extent from MODIS satellite data. Environ. Res. Lett. 2009, 4, 044003. [CrossRef]

14. Romero, R.; Ramis, C.; Guijarro, J.A.; Sumner, G. Daily rainfall affinity areas in Mediterranean Spain. Int. J. Climatol. A J. R. Meteorol. Soc. 1999, 19, 557-578. [CrossRef]

15. Carlson, T.N.; Arthur, S.T. The impact of land use-Land cover changes due to urbanization on surface microclimate and hydrology: A satellite perspective. Glob. Planet. Chang. 2000, 25, 49-65. [CrossRef]

16. Robinson, G.S.; Pigott Burney, L.; Burney, D.A. Landscape paleoecology and megafaunal extinction in southeastern New York State. Ecol. Monogr. 2005, 75, 295-315. [CrossRef]

17. Tatem, A.J.; Noor, A.M.; Hay, S.I. Defining approaches to settlement mapping for public health management in Kenya using medium spatial resolution satellite imagery. Remote Sens. Environ. 2004, 93, 42-52. [CrossRef]

18. Vu, T.T.; Ban, Y. Context-based mapping of damaged buildings from high-resolution optical satellite images. Int. J. Remote Sens. 2010, 31, 3411-3425. [CrossRef]

19. Del Frate, F.; Pacifici, F.; Schiavon, G.; Solimini, C. Use of neural networks for automatic classification from high-resolution images. IEEE Trans. Geosci. Remote Sens. 2007, 45, 800-809. [CrossRef]

20. Strachan, D. Carpow in Context: A Late Bronze Age logboat from the Tay-By David Strachan; The Carpow Logboat: A Bronze Age vessel brought to life. Int. J. Naut. Archaeol. 2011, 40, 204-234. [CrossRef]

21. Mohamed, A.A.F.A.A. Alexandria zero carbon city. Ph.D. Thesis, Faculty of Engineering \& Technology, Arab Academy for Science, Alexandria University, Alexandria Zero Carbon City, Egypt, 14 December 2013.

22. Hemeda, S.; Pitilakis, K.; Bandis, S. Geotechnical, Geophysical Investigations and Seismic Response Analysis of the Underground Tombs in Mustafa Kamil Necropolis. Mediter. Archaeol. Archaeom. 2015, 15, 191-207.

23. Paoletti, V.; Secomandi, M.; Piromallo, M.; Giordano, F.; Fedi, M.; Rapolla, A. Magnetic survey at the submerged archaeological site of Baia, Naples, Southern Italy. Archaeol. Prospect. 2005, 12, 51-59. [CrossRef]

24. Passaro, S.; Barra, M.; Saggiomo, R.; Di Giacomo, S.; Leotta, A.; Uhlen, H.; Mazzola, S. Multi-resolution morpho-bathymetric survey results at the Pozzuoli-Baia underwater archaeological site (Naples, Italy). J. Archaeol. Sci. 2013, 40, 1268-1278. [CrossRef]

25. Fedele, L.; Insinga, D.D.; Calvert, A.T.; Morra, V.; Perrotta, A.; Scarpati, C. 40 Ar/39 Ar dating of tuff vents in the Campi Flegrei caldera (southern Italy): Toward a new chronostratigraphic reconstruction of the Holocene volcanic activity. Bull. Volcanol. 2011, 73, 1323-1336. [CrossRef]

26. Esri. How Spatial Autocorrelation: Moran's I (Spatial Statistics) Works. Available online: http:/ / webhelp.esri.com/arcgisdesktop/9.2/index.cfm?topicname=how_spatial_autocorrelation: moran\%27s_i_(spatial_statistics)_works (accessed on 25 January 2019).

27. Zhu, Y.; Newsam, S. Spatio-temporal sentiment hotspot detection using geotagged photos. In Proceedings of the 24th ACM SIGSPATIAL International Conference on Advances in Geographic Information Systems, Burlingame, CA, USA, 31 October-3 November 2016; p. 76.

28. Wang, Y. Climate Change and Its Ecological and Socioeconomic Impact: Evidence from China's Historical Document for Qing Dynasty. Ph.D. Thesis, Rutgers University, New Brunswick, NJ, USA, 30 May 2010; pp. 1-174.

29. Jindal, S.; Sharma, K. Spatial data mining for finding nearest neighbor and outlier detection. Int. J. Latest Trends Eng. Technol. 2017, 8, 30-36. [CrossRef]

30. Ruda, A. Exploring tourism possibilities using GIS-based spatial association methods. Geogr. Tech. 2016, 11, 87-101. [CrossRef]

31. Marcon, E.; Traissac, S.; Lang, G. A Statistical Test for Ripley's Function Rejection of Poisson Null Hypothesis. ISRN Ecol. 2013, 2013, 753475. [CrossRef] 
32. Sayer, D.; Wienhold, M. A GIS-investigation of four early Anglo-Saxon cemeteries: Ripley's K-function analysis of spatial groupings amongst graves. Soc. Sci. Comput. Rev. 2013, 31, 71-89. [CrossRef]

33. Bjørnstad, O.N.; Ims, R.A.; Lambin, X. Spatial population dynamics: Analyzing patterns and processes of population synchrony. Trends Ecol. Evol. 1999, 14, 427-432. [CrossRef]

34. Sun, W.; Jones, B. Using multi-scale spatial and statistical analysis to assess the effects of brownfield redevelopment on surrounding residential property values in Milwaukee County, USA. Morav. Geogr. Rep. 2013, 21, 56-64. [CrossRef]

(C) 2019 by the authors. Licensee MDPI, Basel, Switzerland. This article is an open access article distributed under the terms and conditions of the Creative Commons Attribution (CC BY) license (http:/ / creativecommons.org/licenses/by/4.0/). 